(C) 2020 Universidad Nacional Autónoma de México, Facultad de Estudios Superiores Zaragoza.

Este es un artículo Open Access bajo la licencia CC BY-NC-ND (http://creativecommons.org/licenses/by-nc-nd/4.0/).

TIP Revista Especializada en Ciencias Químico-Biológicas, 23: 1-17, 2020.

https://doi.org/10.22201/fesz.23958723e.2020.0.266

\title{
Romero (Rosmarinus officinalis L.): su origen, importancia y generalidades de sus metabolitos secundarios
}

\author{
*1Emmanuel Flores-Villa, **Aidé Sáenz-Galindo, \\ Adali Oliva Castañeda-Facio y Rosa Idalia Narro-Céspedes \\ $1 *$ Estudiante de Posgrado en Ciencia y Tecnología Química. Cuerpo \\ Académico de Ciencia y Tecnología de Polímeros. Facultad de Ciencias \\ Químicas. Universidad Autónoma de Coahuila. Boulevard Venustiano \\ Carranza e Ing. José Cárdenas Valdez, Saltillo, 25280, Coahuila, México. \\ E-mails:*emmanuelflores@uadec.edu.mx,**aidesaenz@uadec.edu.mx
}

\begin{abstract}
RESUMEN
El presente trabajo, es una revisión que muestra el impacto que tiene el uso de las plantas medicinales en la salud humana. Desde la antigüedad, por tradición, se extendió el uso mayoritario de algunas familias vegetales como la Lamiaceae que destaca por sus propiedades aromáticas y aplicación culinaria en numerosas culturas, así como en el tratamiento y prevención de una gran variedad de enfermedades y malestares. Dentro de las especies pertenecientes a esta familia se encuentra el romero (Rosmarinus officinalis), planta que ha ganado importancia en el campo de la investigación por sus diversos atributos biológicos como: antiinflamatorio, antimicrobiano, antioxidante $y$ anticancerígeno, entre otros; resultados que debe a sus metabolitos secundarios como: el ácido carnósico, el carnosol, el ácido rosmárico y el alcanfor, entre otros más, aunado a un potencial efecto cuando es aplicado. También se mencionan algunas metodologías que buscan la extracción de los componentes biológicamente activos del romero.

Palabras clave: Lamiaceae, romero (Rosmarinus officinalis L.), metabolito secundario, actividad biológica y extracción.
\end{abstract}

Rosemary (Rosmarinus officinalis L.): its origin, importance and generalities of its secondary metabolites

\begin{abstract}
This paper is a review that shows the impact that the use of medicinal plants has on human health. Since antiquity by tradition the majority use of some plant families was extended as the Lamiaceae that stands out for its aromatic properties and culinary application in many cultures, as well as in the treatment and prevention of a wide variety of diseases and ailments. Among the species belonging to this family is rosemary (Rosmarinus officinalis) a plant that has gained importance in the field of research for its biological attributes such as: anti-inflammatory, antimicrobial, antioxidant and anticancer, among others; results due to its secondary metabolites such as: carnosic acid, carnosol, rosmaric acid and camphor, among others, with a potential effect when applied. Some methodologies that seek the extraction of biologically active components of rosemary are also mentioned.
\end{abstract}

Keywords: Lamiaceae, rosemary (Rosmarinus officinalis L.), secondary metabolite, biological activity and extraction. 


\section{INTRODUCCIÓN}

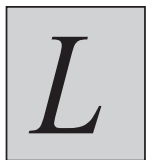

a historia mundial menciona, que para las civilizaciones fue de importancia relevante el uso culinario que dieron a las plantas, así como en la composición de perfumes y medicinas, siendo esta última de uso terapéutico tradicional en todas las culturas (Goudjil et al., 2020). Otros usos son para elaboración de cosméticos, nutracéuticos, agentes anti-edad y colorantes (Benelli \& Oliveira, 2019).

Las plantas son ricas en antioxidantes, enzimas, glutatión, moléculas de naturaleza fenólica y vitaminas, que previenen tanto la auto-oxidación de los triglicéridos insaturados (Nieto, Ros \& Castillo, 2018), como prueba de su efectividad contra el estrés oxidativo en los seres vivos (El-Hadary, Elsanhoty \& Ramadan, 2019), que igual controlan y reducen el daño oxidativo en alimentos, causado por especies reactivas de oxígeno, incrementado así la vida de anaquel y calidad del producto (Altemimi, Lakhssassi, Baharlouei, Watson \& Lightfoot, 2017).

Desde la antigüedad hasta el día de hoy las plantas que poseen aceites esenciales han sido usadas como una fuente de tratamientos profilácticos y medicinales (Shanaida \& Golembiovska, 2018), debido a que contienen moléculas volátiles con actividad biológica, como la antioxidante, antimicrobiana, antiséptica, antiinflamatoria (Goudjil et al., 2020), anticancerígena, analgésica y sedante (Fikry, Khalil \& Salama, 2019).

Dentro de la composición de una gran cantidad de plantas, se encuentra la presencia de diversos metabolitos secundarios, que exhiben un amplio rango de actividades biológicas, hecho que ha influido en el actual incremento de las investigaciones enfocadas en determinar cuáles son los componentes principales que otorgan estas propiedades (Milevskaya, Prasad \& Temerdashev, 2019), los metabolitos se encuentran principalmente en el aceite esencial y en los extractos de las plantas, que reportan actividades antimicrobianas, antiinflamatorias y antioxidantes, así como hipolipemiantes (Fikry et al., 2019). Cabe destacar que los metabolitos secundarios son el producto de las reacciones enzimáticas de las plantas, con funciones de atracción, defensa o señalización (Khaw, Parat, Shaw \& Falconer, 2017).

La presencia de estas moléculas en las plantas varía, principalmente, según el área geográfica donde se encuentren y a sus condiciones de crecimiento (Fikry et al., 2019; Zinicovscaia et al., 2020). Otro factor que afecta la producción de estos metabolitos es el estrés generado por la sequía, las plantas reducen la biosíntesis de estas moléculas, cambiando la composición química de sus aceites esenciales y extractos, es decir de sus metabolitos secundarios en general (García et al., 2019).
En el contexto de las plantas medicinales, se destaca la familia Lamiaceae, sexta de las plantas aromáticas, más abundante y una de las más extensas dentro de las dicotiledóneas (García et al., 2019), posee una elevada popularidad por el potencial aromático de la mayoría de sus especies (Gürbüz et al., 2019). Dentro de la familia Lamiaceae destaca la importancia del género Rosmarinus, que contiene las siguientes especies: $R$. laxiflorus, $R$. eriocalyx, $R$. tomentosus, $R$. lavandulaceus y $R$. officinalis, siendo esta última la más utilizada, gracias a que sus metabolitos secundarios se encuentran en casi todas las partes de la planta (hojas, tallos, flores y raíces) mostrando éstas una gran variedad de actividades biológicas benéficas (Ali, Chua \& Chow, 2019).

Es por ello, que el objetivo principal de este artículo de revisión es enfatizar la importancia del romero Rosmarinus officinalis L. (R. officinalis L.) dentro de la familia Lamiaceae, su valor económico las propiedades biológicas y aplicaciones de sus metabolitos secundarios, así como las generalidades en torno a su extracción. La revisión de la literatura, se hizo consultando en las bases de datos de Nature, SciFinder, Scopus y SpringerLink, utilizando como palabras clave: "Lamiaceae", "rosemary", "Rosmarinus officinalis", "techniques for extraction of natural products" y "green extraction technologies".

\section{Familia Lamiaceae}

En el Orden de las Lamiales la familia Lamiaceae (también conocida como Labiatae) (Fidan et al., 2019) es la más grande de todas, con alrededor de 236 géneros y de 6, 900 a 7, 200 especies (Karpiński, 2020; Fidan et al., 2019) repartidas en todo el mundo, especialmente en la región del Mediterráneo y el suroeste de Asia (Zinicovscaia et al., 2020). Los géneros con mayor cantidad de especies pertenecen a Salvia con 900, Scutellaria con 360, Stachys y Plectranthus con 300, Hyptis con 280, Teucrium y Vitex con 250, Thymus con 220 y Nepeta con 200 (Karpiński, 2020).

Algunos de los miembros de esta familia que poseen importancia económica como plantas culinarias son el tomillo, la albahaca, el romero, la menta, el orégano, la salvia, la hierbabuena (Gürbüz et al., 2019), la lavanda, el hisopo, la mejorana y el toronjil (Sik, Kapcsándi, Székelyhidi, Hanczné \& Ajtony, 2019), que tienen un elevado contenido de moléculas aromáticas (Fidan et al., 2019).

La Figura 1 muestra algunas de las plantas que forman parte de la familia Lamiaceae, un gran número de ellas con propiedades aromáticas (permitiendo su uso como especias) y relativa facilidad para ser cultivadas (Sik et al., 2019; Risaliti et al., 2019) presentan estructuras glandulares externas productoras de aceites volátiles, que las hace importantes y de gran valor para la industria alimenticia, cosmética y farmacéutica (García et al., 2019), así como en la agricultura, 


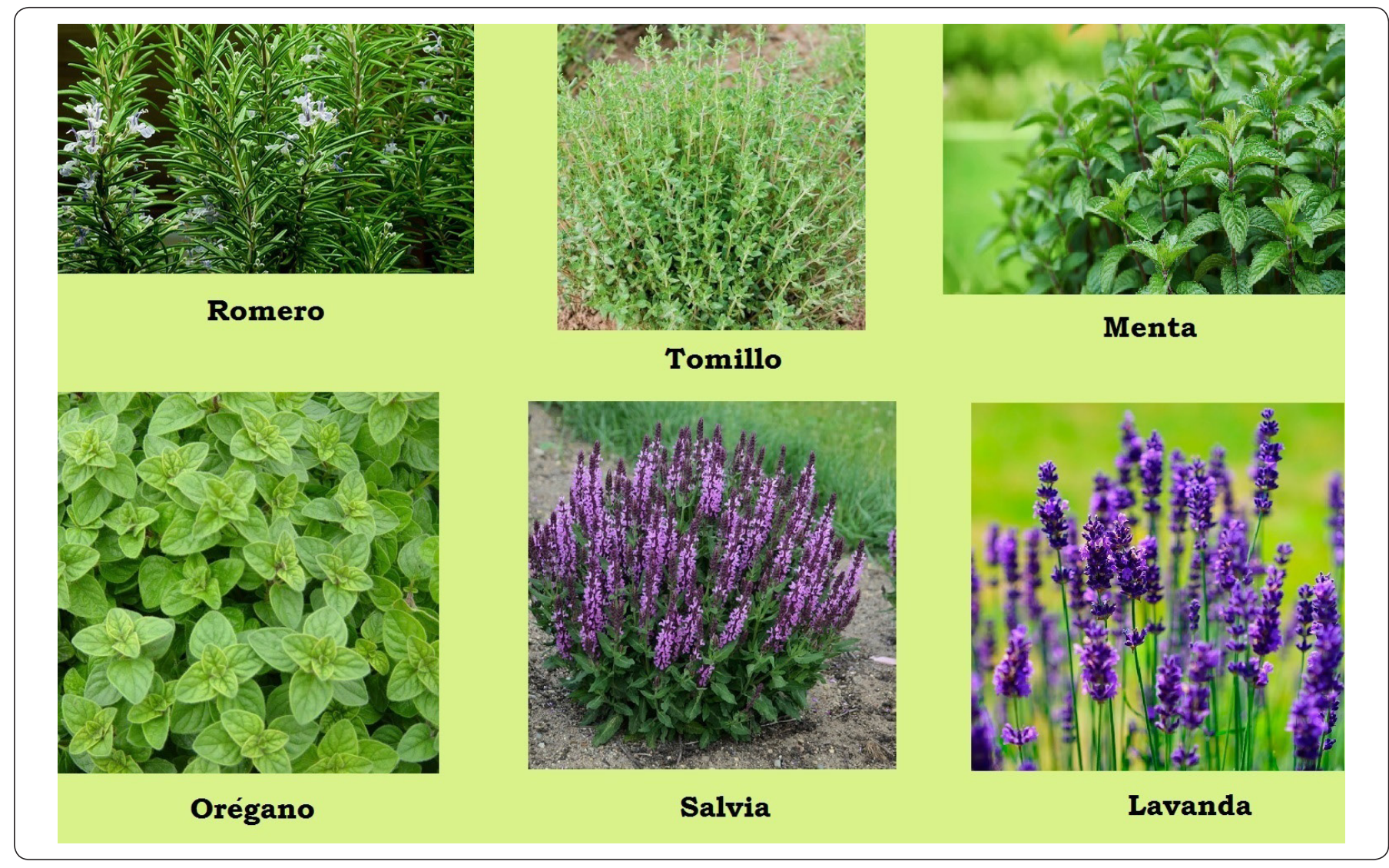

Figura 1. Ejemplos de algunas plantas pertenecientes a la familia Lamiaceae. NOTA: Fotografías tomadas de internet cuyas direcciones son las siguientes: Romero: https://bit.ly/312Z7MD. Página "ASAJA-JAÉN" organización profesional agraria. Tomillo: https://bit. ly/3fbHqja. Página de la comunidad "FLICKR" por Rafael de Jésus. Menta: https://bit.ly/2CTtWeR. Página del blog "Comer con conciencia: Alimente" por Verónica Mollejo. Orégano: https://bit.ly/2P6KwKH. Página del blog de ciencia "Bajo el microscopio" por Miguel Verde. Salvia: https://bit.ly/3jOLJ7o. Página de venta de plantas y accesorios de jardín "Beechmount Garden Centre". Lavanda: https://bit.ly/2DkAzXr. Página de venta de semillas "Entre Semillas".

demostrando sus propiedades insecticidas, en el control de plagas (Fotso et al., 2019).

Una de las principales características de la familia, es la producción de aceite esencial en los géneros de la subfamilia Nepetoideae destacando Rosmarinus, Mentha, Lavandula, Thymus y Origanum (Bridi, de Loreto, Anders \& Von Poser, 2020).

Las diversas especies de la familia Lamiaceae muestran un alto contenido de fenoles como los polifenoles, cumarinas, quinonas, diterpenoides, triterpenoides, iridoides, saponinas y en algunos casos alcaloides pirrolidínicos y piridínicos. (Lemjallad et al., 2019). Una gran cantidad de estos componentes se relaciona con el elevado poder antioxidante que ostentan estas plantas (Brown, John \& Shahidi, 2019).

Esta familia se considera como medicinal, por lo que su uso es muy activo en la fitoterapia (Milevskaya et al., 2019).
La presencia de aceites esenciales con moléculas volátiles biológicamente activas en estas hierbas, ha permitido su aplicación en la aromaterapia (Romero, Ramasamy, Meng, Abdul \& Agatonovic-Kustrin, 2019), además de ser usados en la medicina tradicional y moderna, como fuente de medicinas terapéuticas y profilácticas (Shanaida \& Golembiovska, 2018), sus extractos y aceites esenciales presentan elevadas propiedades antioxidantes y antimicrobianas (Goudjil et al., 2020), siendo esta última aprovechada en la industria alimenticia contra microorganismos patógenos y causantes del deterioro en alimentos (Tančinová, Medo, Mašková, Foltinová \& Árvay, 2019).

La importancia medicinal de la familia Lamiaceae se debe principalmente a la presencia de sustancias bioactivas en la mayoría de las especies, lo cual brinda beneficios en el tratamiento de enfermedades gástricas, respiratorias y nerviosas, así como en la prevención de la diabetes (Ruzzene, Sete, Botelho \& Alberton, 2019). En la medicina tradicional 
también han sido usadas como tratamiento para el asma, depresión, estrés, dolor de cabeza y Alzheimer (Sik et al., 2019). Algunos estudios evidencian un posible efecto preventivo o terapéutico en enfermedades cardiovasculares, problemas de obesidad, desórdenes neurodegenerativos y cáncer (Sik, Hanczné, Kapcsándi \& Ajtony, 2020).

En la medicina tradicional y moderna, las aplicaciones de las plantas de la familia Lamiaceae son muy variadas, debido a la presencia de diversos metabolitos, componentes principales de los aceites esenciales como los ácidos orgánicos, saponinas y taninos, entre otros, que poseen propiedades antifúngicas, antiinflamatorias, antimicrobianas, antioxidantes, antibacterianas (Zinicovscaia et al., 2020), antialérgicas, astringentes, anticarcinogénicas (Sik et al., 2019), hepatoprotectoras, antivirales, antitumorales (Shanaida \& Golembiovska, 2018), insecticidas y acaricidas (Lemjallad et al., 2019).

\section{ROMERO (R. officinalis L.)}

Una de las plantas de mayor importancia económica dentro de la familia Lamiaceae es el romero $R$. officinalis (ElDesouky, Mahmoud, Riad \& Taha, 2019), también conocido por los sinónimos de Salvia rosmarinus (nombre actualmente aceptado) y Rosmarinus angustifolius (Borges, Sánchez, Matias, Keita \& Tavares, 2018), el nombre del romero deriva de las palabras del latín "ros" y "marinus" lo cual se interpreta como "rocío del mar" o "rocío marino" (Fidan et al., 2019). En Europa y Estados Unidos de América es conocido como "rosemary" y en Brasil como "Alecrim" (Amaral et al., 2017).

El romero es una planta de hojas fragantes, perennes y de color verde (Basheer, 2018), con flores azul blanquecinas (Ali et al., 2019), nativa del Mediterráneo, del norte y sur de África, así como de Asia Occidental (Karadağ et al., 2019), crece en muchos lugares del planeta (en suelos secos o moderadamente húmedos), alcanzando una altura de 1 a 2 metros. No tolera suelos anaeróbicos o muy mojados, pero sí los de salinidad media. Su período de floración es de mayo a junio y el de fructificación es de primavera a verano (Borges et al., 2018).

Los principales usos del romero son en cosméticos, saborizantes de comida (Basheer, 2018; Borges et al., 2018), mejoradores de la vida de anaquel en alimentos perecederos (Fidan et al., 2019; Said, Waheed \& Khalifa, 2019), estimulantes del crecimiento del cabello, como aroma en la preparación de perfumes y fragancias (Endo et al., 2018), como aditivo para shampoo, crema y jabón (Akhbari, Masoum, Aghababaei \& Hamedi, 2018), en colorantes naturales de lociones y enjuagues bucales (Gomes et al., 2020).

En la medicina tradicional se ha usado en el tratamiento del asma bronquial, la epilepsia, el dolor de cabeza, malestares gastrointestinales, cólicos biliares y renales (Said et al.,
2019), el control de alergias, pérdida del apetito, anomalías circulatorias, complemento en el tratamiento del dolor muscular, de articulaciones y en inflamaciones (Borges et al., 2018), también como antiespasmódico, carminativo, diurético, antirreumático, antidepresivo, ansiolítico, potenciador de la fertilidad humana y la memoria (Karim, Khan, Abdelhalim, Abdel-Halim \& Hanrahan, 2017), en el tratamiento de enfermedades inflamatorias y de la diabetes mellitus (Nieto et al., 2018), potenciador de la cognición y de la circulación sanguínea (Sadeh et al., 2019), expectorante, en el tratamiento de la dismenorrea, desórdenes respiratorios, dolor de garganta y de estómago (Karadağ et al., 2019), en desórdenes de la piel (Ali et al., 2019), el alivio de enfermedades cardiovasculares (Zhang et al., 2019), el tratamiento de lesiones orales (Sumintarti, Fatimasari, Hajrah-Yusuf \& Ruslin, 2018), como atenuante de cataratas, tratamiento de alergias cutáneas (de Oliveira, Camargo \& de Oliveira, 2019), como tónico en el tratamiento de las flatulencias y tensión nerviosa (Abbaszadeh, Layeghhaghighi, Azimi \& Hadi, 2020) y control de la caspa (Trupti \& Gadekar, 2018).

Las aplicaciones del romero se dan principalmente en las industrias: alimenticia, fragancias (Fidan et al., 2019), producción orgánica de peces y caviar (Ebrahimi, Haghjou, Nematollahi \& Goudarzian, 2020; Zoral et al., 2018), cosmética (Sadeh et al., 2019), farmacéutica (Benelli \& Oliveira, 2019), avícola (Abo, Elsadek, Taha, Abd \& ElSabrout, 2020) y pieles como el cuero (Gomes et al., 2020).

$\mathrm{Su}$ aceite esencial y extractos son antimicrobianos con propiedades antioxidantes (Basheer, 2018), anti-nefrotóxicos, antiinflamatorios, antitumorales, anti-hepatotóxicos (ElDesouky et al., 2019), antihelmínticos (Zoral et al., 2018), anticancerígenos (Karim et al., 2017), antifúngicos (Ebrahimi et al., 2020), insecticidas, antidiabéticos, antimutagénicos, anti-toxigénicos (Nieto et al., 2018), citotóxicos, analgésicos, herbicidas, hipoglucémicos, hipolipemiantes (Karadağ et al., 2019), antiartríticos (Beltrán et al., 2017), antidepresivos, antiobesidad, neuroprotectores (Hamidpour, Hamidpour \& Elias, 2017), antihiperglucémicos, antihiperlipidémicos (Ali et al., 2019), antidiarreicos (Selmi, Rtibi, Grami, Sebai \& Marzouki, 2017) e inhibidoreres de la germinación de semillas (Sadeh et al., 2019), efecto inhibidor contra el VIH (Barbieri et al., 2019), vasorrelajantes (Zhang et al., 2019), antivirales (Sumintarti et al., 2018), antitrombóticos (Elyemni et al., 2019), hipouricémicos, proapoptóticos (de Oliveira et al., 2019), anti-infecciosos, espasmolíticos (Gomes et al., 2020), entre otros más.

Una gran cantidad de investigaciones se centran hoy en día, en el estudio del romero, destacando aquellas que han demostrado los efectos farmacológicos de sus moléculas fenólicas contra procesos inflamatorios, cardiopatías isquémicas, ateroesclerosis, úlceras gástricas, deficiencias 
respiratorias y algunos tipos de cáncer (Amaral et al., 2017). Se ha encontrado también que mejora la producción del factor de crecimiento nervioso (Karim et al., 2017).

El potencial antioxidante de los extractos de romero ha permitido su uso de forma regular en el control de la oxidación de lípidos presentes en alimentos (Brown et al., 2019), al interrumpir las reacciones en cadena de los radicales libres (Huang et al., 2020), lo que permite su aplicación como preservativo natural en alimentos cocinados (El-Desouky et al., 2019; Huang et al., 2020).

\section{Metabolitos SECUndarios DEL ROMERo $(R$. officinalis L.)}

Los componentes que confieren las propiedades farmacológicas del romero pueden agruparse en las categorías de flavonoides, terpenoides (monoterpenos, sesquiterpenos, diterpenos, triterpenos) y derivados hidroxicinámicos (Ali et al., 2019).

En el romero se encuentran dos principales constituyentes activos: los flavonoides (como la diosmetina, diosmina, hispidulina, apigenina, luteolina y sinensetina, entre otros) y di y triterpenoides (como la rosmariquinona, el ácido carnósico, ácido ursólico, ácido oleanólico y la picrosalvina, entre otros). Sus moléculas fenólicas pueden atrapar los radicales libres e incrementar de forma indirecta la producción de antioxidantes celulares endógenos (El-Desouky et al., 2019). La actividad antimicrobiana de los extractos y aceite esencial del romero es atribuida principalmente al $\alpha$-pineno, el acetato de bornilo, el alcanfor y el 1,8-cineol (Ebrahimi et al., 2020).

El romero posee alrededor de un $0.5-2.5 \%$ de aceite esencial, conformado en su mayor parte por 1,8-cineol (15-50\%), alcanfor (15-25\%), $\alpha$-pineno (10-25\%), canfeno (5.2-8.6\%) y borneol (3.2-7.7\%) (Sumintarti et al., 2018). El aceite esencial de mayor calidad es obtenido de sus hojas, donde se encuentra la mayoría de las tricomas glandulares que lo secretan (Elyemni et al., 2019).

El ácido rosmárico es uno de los componentes fenólicos más comunes en las plantas pertenecientes a la familia Lamiaceae (Sik et al., 2020), cuyas propiedades y actividad biológica se deben especialmente a la presencia de este metabolito (Sik et al., 2019).

Se ha reportado que en los extractos de romero alrededor del $24 \%$ de las moléculas volátiles que contiene pertenecen a los terpenos (como la verbenona), acetato de bornilo, alcanfor y $\alpha / \beta$ cariofileno (Sadeh et al., 2019), se ha identificado una composición mayor de $\alpha$-pineno, borneol y 1,8-cineol (Karadağ et. al., 2019). Contiene rosmanol, isorosmanol, rosmadial y metil carnosato moléculas con propiedad antioxidante (Quintana, Villanueva, Reglero, García \& Fornari,
2019). También se ha aislado la salvigenina y la cirsimaritina (Karim et al., 2017), así como alcoholes triterpénicos, ácido oleanólico, rosmaquinonas (Beltrán et al., 2017). Otros metabolitos de importancia encontrados son también el ácido ursólico y betulínico (Benelli \& Oliveira, 2019). La Figura 2 muestra las estructuras de algunos metabolitos secundarios presentes en el romero.

El poder preservativo y terapéutico del romero reside en su aceite esencial y extractos, cuya composición química varía dependiendo de las condiciones ecológicas en las que se desarrollan y sin embargo, todos ellos contienen componentes de actividad biológica, como las moléculas fenólicas (ácido carnosínico, carnosol y ácido rosmárico), responsables de la fuerte actividad antioxidante, aprovechada en la preservación de alimentos y en la terapéutica de mecanismos anticáncer $\mathrm{y}$ antidiabetes, convirtiendo al romero en una planta de gran interés en las industrias médica y de alimentos (Hamidpour et. al., 2017). En la Tabla I se resume a los principales metabolitos secundarios encontrados en el romero así como algunas de sus propiedades biológicas y aplicaciones.

\section{EXTRACCIÓN DE METABOLITOS SECUNDARIOS DEL ROMERO (R. officinalis L.)}

Los materiales herbales contienen importantes componentes bioactivos, cuyo aislamiento y purificación se da mediante la extracción como un primer paso de recuperación, descrito como un fenómeno de transporte donde los componentes de interés en la matriz son transferidos a un disolvente adecuado a lo que se busca obtener (Moreira, Alexandre, Pintado \& Saraiva, 2019).

Existen numerosas metodologías para la extracción de las moléculas de actividad biológica de las plantas, que se dividen en dos grupos: técnicas convencionales y técnicas no convencionales (Sik et al., 2020; Debebe, Shimelis, Asfaw \& Jong, 2019).

En las técnicas convencionales se encuentran las de extracción por reflujo, percolación y maceración, que utilizan disolventes orgánicos en elevadas cantidades, así como largos tiempos de extracción (Zhang, Lin \& Ye, 2018). También se encuentran el prensado mecánico y la trituración, con inconvenientes como baja eficiencia y severas condiciones de extracción, pobre selectividad y el uso de disolventes orgánicos volátiles (Ventura et al., 2017). Los disolventes utilizados en estas extracciones son un factor importante porque pueden afectar la eficiencia de la extracción de los componentes bioactivos y sus posteriores beneficios en la salud (Ngo, Scarlett, Bowyer, Ngo \& Voung, 2017), además de considerar que en la aplicación de las metodologías el uso de disolventes orgánicos es necesario, evitando el uso de aquellos que son tóxicos por los residuos que quedan en el producto final (Pantoja, Hurtado \& Martínez, 2017). 
<smiles>CC(C)c1cc2c(c(O)c1O)[C@H]1CCCC(C)(C)[C@H]1CC2</smiles>

Ácido carnósico<smiles>CC(C)c1cc2c(c(O)c1O)[C@@]13CCCC(C)(C)[C@H]1C[C@H]2OC3=O</smiles><smiles>O=C(/C=C/c1ccc(O)cc1)O[C@@H](Cc1ccc(O)c(O)c1)C(=O)O</smiles>

Ácido rosmárico

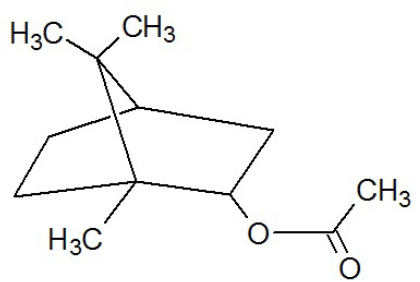

Acetato de bornilo

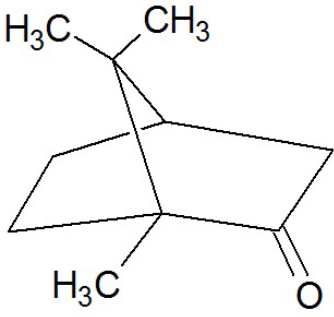

Alcanfor

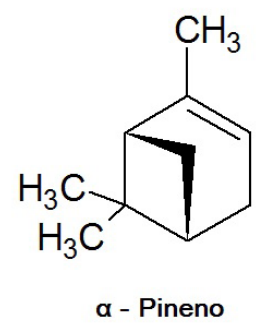

Figura 2. Estructuras de algunos metabolitos secundarios presentes en el romero (Rosmarinus officinalis L.). NOTA: Estructuras elaboradas con el software ACD/ChemSketch (Freeware), por Emmanuel Flores Villa. Versión 11.02 (Build 25941, 21 may 2008).

Las técnicas no convencionales buscan superar las limitaciones de las extracciones convencionales con rendimientos de extracción más altos y ahorro de energía (Putnik et al., 2017). Entre las metodologías de esta categoría encontramos las extracciones asistidas por microondas, enzimas, con fluidos supercríticos (Sik et al., 2020), ultrasonido (Debebe et al., 2019), con líquidos iónicos (Ventura et al., 2017), por campos eléctricos pulsantes, tratamientos hidrotérmicos a altas temperaturas e hidrólisis alcalina (Jesus et al., 2019), entre otras.

En la Tabla II se muestra un comparativo de las tecnologías convencionales y las no convencionales más utilizadas para la extracción de los componentes bioactivos de las plantas.

Los extractos del romero que actualmente están en el mercado, se obtienen por secado y molido de sus hojas, seguido de una extracción usando disolventes como la acetona, el etanol, el metanol, el hexano y agua, o mezclas de ellos. El producto resultante puede ser filtrado, para remover los residuos sólidos, seguido de una evaporación de disolventes al vacío, secado por pulverización y tamizado para obtener un fino polvo. Un paso más, incluye una desodorización y/o decoloración usando excipientes grado alimenticio (Senanayake, 2018), como el almidón, empleado en la industria farmacéutica (Sullbarán, Matiz \& Baena, 2018).
En el laboratorio, la técnica más utilizada para la extracción de metabolitos secundarios del romero es la maceración. La obtención de extractos acuosos del romero mediante la maceración de sus hojas con agua destilada, requiere un adecuado control de la temperatura con el fin de evitar el crecimiento de microorganismos (Zoral et al., 2018). Se opta por usar una solución hidroalcohólica como agente extractor en las hojas del romero (Endo et al., 2018). También se trabaja con sus flores como materia prima (Karadağ et al., 2019). El prensado en frío es una técnica usada en la obtención del aceite esencial de hojas de romero (El-Hadary et al., 2019). Las técnicas de extracción convencionales, asistidas con tecnologías como el ultrasonido, microondas, extracción con líquido presurizado o usando $\mathrm{CO}_{2}$ supercrítico son algunas formas de mejorar la extracción de los metabolitos secundarios del romero (Quintana et al., 2019).

La obtención del extracto de romero asistido por fluidos supercríticos, se lleva a cabo usando sus hojas secas, utilizando $\mathrm{CO}_{2}$ supercrítico como disolvente extractor (Senanayake, 2018).

El uso del ultrasonido para las extracciones de los componentes de interés del romero, incrementa la eficiencia en la obtención de los compuestos bioactivos (Hosseinie, Bolourian, Yaghoubi \& Ghanuni, 2018). 


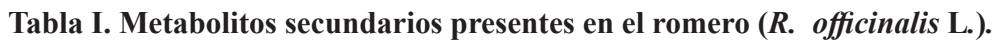

\begin{tabular}{|c|c|c|c|}
\hline \multicolumn{4}{|c|}{ Metabolitos presentes en el romero } \\
\hline Metabolitos & Actividad biológica & Usos/aplicaciones & Referencias \\
\hline Ácido carnósico. & $\begin{array}{l}\text { Antioxidante, antimicrobiana, } \\
\text { hepatoprotectora, } \\
\text { hipoglucémica, } \\
\text { hipolipemiante, } \\
\text { anticáncer, vasorrelajante, } \\
\text { antiinflamatoria y } \\
\text { antitumoral. }\end{array}$ & $\begin{array}{l}\text { Control de la oxidación en } \\
\text { los alimentos, conservador } \\
\text { de alimentos preparados y } \\
\text { como tónico en el alivio de la } \\
\text { circulación. }\end{array}$ & $\begin{array}{l}\text { (El-Desouky et al., 2019) (Brown et al., } \\
\text { 2019) (Huang et al., 2020) (Hamidpour et } \\
\text { al., 2017) (Basheer, 2018) (Said et al., 2019) } \\
\text { (Ebrahimi et al., 2020) (Nieto et al., 2018) } \\
\text { (Karadağ et al., 2019) (Quintana et al., 2019) } \\
\text { (Beltrán et al., 2017) (Benelli \& Oliveira, } \\
\text { 2019) (Zhang et al., 2019) (de Oliveira et al., } \\
\text { 2019). }\end{array}$ \\
\hline Carnosol. & $\begin{array}{l}\text { Antioxidante, antimicrobiana, } \\
\text { hepatoprotectora, } \\
\text { hipoglucémica, } \\
\text { hipolipemiante, } \\
\text { anticáncer, vasorrelajante, } \\
\text { antiproliferativa, antifúngica, } \\
\text { antinflamatoria y } \\
\text { antidiabética. }\end{array}$ & $\begin{array}{l}\text { Control de la oxidación en } \\
\text { los alimentos, conservador de } \\
\text { alimentos preparados, como } \\
\text { tónico en la mejora de la } \\
\text { circulación y como aditivo en } \\
\text { el tratamiento de la caspa. }\end{array}$ & $\begin{array}{l}\text { (El-Desouky et al., 2019) (Brown et al., } \\
\text { 2019) (Huang et al., 2020) (Hamidpour et } \\
\text { al., 2017) (Basheer, 2018) (Said et al., 2019) } \\
\text { (Ebrahimi et al., 2020) (Nieto et al., 2018) } \\
\text { (Karadağ et al., 2019) (Quintana et al., 2019) } \\
\text { (Beltrán et al., 2017) (Benelli \& Oliveira, } \\
\text { 2019) (Ali et al., 2019) (Zhang et al., 2019) } \\
\text { (de Oliveira et al., 2019). }\end{array}$ \\
\hline Ácido rosmárico. & $\begin{array}{l}\text { Antioxidante, antimicrobiana, } \\
\text { hepatoprotectora, } \\
\text { hipoglucémica, } \\
\text { hipolipemiante, anticáncer, } \\
\text { neuroprotectora, } \\
\text { antiproliferativa y antiviral. }\end{array}$ & $\begin{array}{l}\text { Control de la oxidación en los } \\
\text { alimentos y conservador de } \\
\text { alimentos preparados. }\end{array}$ & $\begin{array}{l}\text { (Ebrahimi et al., 2020) (Brown et al., 2019) } \\
\text { (Huang et al., 2020) (Hamidpour et al., 2017) } \\
\text { (Basheer, 2018) (Karim et al., 2017) (Said et } \\
\text { al., 2019) (Karadağ et al., 2019) (Quintana } \\
\text { et al., 2019) (Beltrán et al., 2017) (Benelli \& } \\
\text { Oliveira, 2019) (Ali et al., 2019). }\end{array}$ \\
\hline Rosmaridifenol. & Antioxidante. & $\begin{array}{l}\text { Control de la oxidación en los } \\
\text { alimentos. }\end{array}$ & (Huang et al., 2020). \\
\hline Ácido ursólico. & $\begin{array}{l}\text { Antioxidante, citotóxica, } \\
\text { anticancerígena, } \\
\text { hipouricémico y } \\
\text { proapoptótica. }\end{array}$ & $\begin{array}{l}\text { Control de la oxidación en los } \\
\text { alimentos y como tónico en el } \\
\text { control del ácido úrico. }\end{array}$ & $\begin{array}{l}\text { (Huang et al., 2020) (Benelli \& Oliveira, } \\
\text { 2019) (El-Desouky et al., 2019) (Basheer, } \\
\text { 2018) (Beltrán et al., 2017) (Ali et al., 2019) } \\
\text { (de Oliveira et al., 2019). }\end{array}$ \\
\hline$\beta$-pineno. & Antiparasitaria e insecticida. & $\begin{array}{l}\text { Tratamiento de enfermedades } \\
\text { parasitarias en peces, } \\
\text { promotor del crecimiento e } \\
\text { inmunoestimulante en peces } \\
\text { y en el control de plagas en la } \\
\text { agricultura. }\end{array}$ & $\begin{array}{l}\text { (Borges et al., 2018) (Zoral et al., 2018) } \\
\text { (Ebrahimi et al., 2020) (Ali et al., 2019) } \\
\text { (Selmi et al., 2017) (Kowalski et al., 2018) } \\
\text { (Sadeh et al., 2019) (Ferreira et al., 2020). }\end{array}$ \\
\hline Acetato de bornilo. & $\begin{array}{l}\text { Antimicrobiana, } \\
\text { insecticida, antioxidante, } \\
\text { hepatoprotectora, } \\
\text { hipoglucémica, } \\
\text { hipolipemiante, anticáncer y } \\
\text { antifúngico. }\end{array}$ & $\begin{array}{l}\text { Promotor del crecimiento } \\
\text { e inmunoestimulante en } \\
\text { peces, control de plagas en } \\
\text { la agricultura, así como en } \\
\text { el control y tratamiento de la } \\
\text { caspa. }\end{array}$ & $\begin{array}{l}\text { (Ebrahimi et al., 2020) (Sadeh et al, 2019) } \\
\text { (Karadağ et al., 2019) (Ali et al., 2019) } \\
\text { (Selmi et al., 2017) (Kowalski et al., 2018) } \\
\text { (Elbahnasawy et al., 2019) (Trupti \& } \\
\text { Gadekar, 2018). }\end{array}$ \\
\hline
\end{tabular}


Tabla I. Metabolitos secundarios presentes en el romero (R. officinalis L.) (continuación).

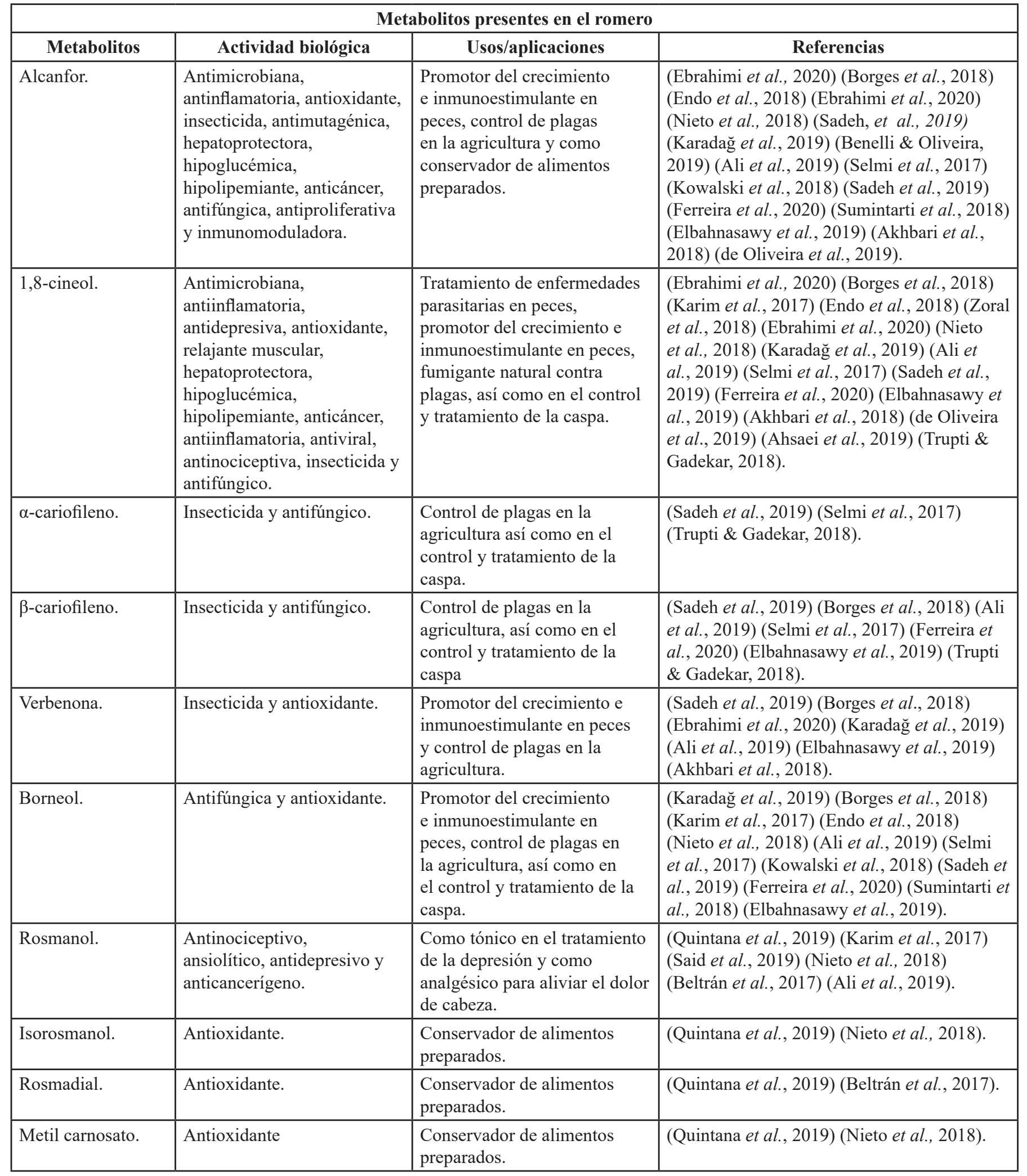


Tabla I. Metabolitos secundarios presentes en el romero ( $R$. officinalis L.) (continuación).

\begin{tabular}{|c|c|c|c|}
\hline \multicolumn{4}{|c|}{ Metabolitos presentes en el romero } \\
\hline Metabolitos & Actividad biológica & Usos/aplicaciones & Referencias \\
\hline Salvigenina. & $\begin{array}{l}\text { Antinociceptiva, ansiolítica y } \\
\text { antidepresiva. }\end{array}$ & $\begin{array}{l}\text { Como tónico en el tratamiento } \\
\text { de la depresión y como } \\
\text { analgésico para aliviar el } \\
\text { dolor de cabeza, garganta y } \\
\text { estómago. }\end{array}$ & (Karim et al., 2017). \\
\hline Ácido oleanólico. & $\begin{array}{l}\text { Antiviral, antitumoral, } \\
\text { antioxidante y } \\
\text { antiproliferativa. }\end{array}$ & $\begin{array}{l}\text { Conservador de alimentos } \\
\text { preparados y suplemento } \\
\text { nutricional. }\end{array}$ & $\begin{array}{l}\text { (Beltrán et al., 2017) (El-Desouky et al., } \\
\text { 2019) (Ali et al., 2019) (de Oliveira et al., } \\
\text { 2019). }\end{array}$ \\
\hline Ácido betulínico. & Antioxidante. & $\begin{array}{l}\text { Conservador de alimentos } \\
\text { preparados. }\end{array}$ & (Benelli \& Oliveira, 2019) (Ali et al., 2019). \\
\hline Limoneno. & $\begin{array}{l}\text { Anticancerígena y } \\
\text { hepatoprotectora. }\end{array}$ & $\begin{array}{l}\text { Como tónico coadyuvante en } \\
\text { tratamientos del cáncer. }\end{array}$ & $\begin{array}{l}\text { (Borges et al., 2018) (Karadağ et al., 2019) } \\
\text { (Ali et al., 2019) (Kowalski et al., 2018) } \\
\text { (Sadeh et al., 2019) (Elbahnasawy et al., } \\
\text { 2019) (Elyemni et al., 2019). }\end{array}$ \\
\hline Mirceno. & $\begin{array}{l}\text { Analgésica, antibacteriana y } \\
\text { antioxidante. }\end{array}$ & $\begin{array}{l}\text { Promotor del crecimiento e } \\
\text { inmunoestimulante en peces. }\end{array}$ & $\begin{array}{l}\text { (Borges et al., 2018) (Ebrahimi et al., 2020) } \\
\text { (Ali et al., 2019) (Kowalski et al., 2018) } \\
\text { (Ferreira et al., 2020) (Elbahnasawy et al., } \\
\text { 2019) (Abbaszadeh et al., 2020). }\end{array}$ \\
\hline Ácido cafeico. & $\begin{array}{l}\text { Antibacteriana, antioxidante } \\
\text { y antitumoral. }\end{array}$ & $\begin{array}{l}\text { Control de la oxidación en } \\
\text { alimentos y conservador de } \\
\text { alimentos preparados. }\end{array}$ & $\begin{array}{l}\text { (Basheer, 2018) (Nieto et al., 2018) (Beltrán } \\
\text { et al., 2017) (Benelli \& Oliveira, 2019) (Ali } \\
\text { et al., 2019) (Sumintarti et al., 2018) (de } \\
\text { Oliveira et al., 2019) (Gomes et al., 2020). }\end{array}$ \\
\hline$\alpha$-terpineol. & Analgésica y antioxidante. & $\begin{array}{l}\text { Como tónico en el alivio del } \\
\text { dolor de cabeza, garganta y } \\
\text { estómago. }\end{array}$ & $\begin{array}{l}\text { (Borges et al., 2018) (Karim et al., 2017) } \\
\text { (Ali et al., 2019) (Kowalski et al., 2018) } \\
\text { (Sadeh et al., 2019) (Ferreira et al., 2020) } \\
\text { (Elbahnasawy et al., 2019) (Elyemni et al., } \\
\text { 2019). }\end{array}$ \\
\hline Terpinoleno. & Antifúngica y antibacteriana. & $\begin{array}{l}\text { Aditivo aromático en jabones } \\
\text { y como tratamiento en el } \\
\text { control de la caspa. }\end{array}$ & $\begin{array}{l}\text { (Borges et al., 2018) (Ali et al., 2019) } \\
\text { (Elbahnasawy et al., 2019). }\end{array}$ \\
\hline Galocatequina. & Antioxidante. & $\begin{array}{l}\text { Control de la oxidación en } \\
\text { alimentos y conservador de } \\
\text { alimentos preparados. }\end{array}$ & (Karim et al., 2017). \\
\hline
\end{tabular}


Tabla I. Metabolitos secundarios presentes en el romero (R. officinalis L.) (continuación).

\begin{tabular}{|c|c|c|c|}
\hline \multicolumn{4}{|c|}{ Metabolitos presentes en el romero } \\
\hline Metabolitos & Actividad biológica & Usos/aplicaciones & Referencias \\
\hline$P$-cimeno. & Antioxidante. & $\begin{array}{l}\text { Promotor del crecimiento e } \\
\text { inmunoestimulante en peces. }\end{array}$ & $\begin{array}{l}\text { (Ebrahimi et al., 2020) (Ali et al., 2019) } \\
\text { (Kowalski et al., 2018) (Sadeh et al., 2019) } \\
\text { (Elyemni et al., 2019) (Abbaszadeh et al., } \\
\text { 2020). }\end{array}$ \\
\hline Diosmina. & Antiinflamatoria. & $\begin{array}{l}\text { Ayuda en el alivio de la } \\
\text { circulación periférica. }\end{array}$ & $\begin{array}{l}\text { (Nieto et al., 2018) (El-Desouky et al., 2019) } \\
\text { (Ali et al., 2019) (Sumintarti et al., 2018). }\end{array}$ \\
\hline Hispidulina. & $\begin{array}{l}\text { Antioxidantes y } \\
\text { antiinflamatorias. }\end{array}$ & $\begin{array}{l}\text { Conservador de alimentos } \\
\text { preparados. }\end{array}$ & (Nieto et al., 2018) (El-Desouky et al., 2019). \\
\hline Luteolina. & $\begin{array}{l}\text { Antiinflamatoria, } \\
\text { antimicrobiana y } \\
\text { antiproliferativa. }\end{array}$ & $\begin{array}{l}\text { Conservador de alimentos } \\
\text { preparados. }\end{array}$ & $\begin{array}{l}\text { (Karadağ et al., 2019) (El-Desouky et al., } \\
\text { 2019) (Ali et al., 2019) (Sumintarti et al., } \\
\text { 2018). }\end{array}$ \\
\hline Apigenina. & $\begin{array}{l}\text { Antioxidante y } \\
\text { antibacteriana. }\end{array}$ & $\begin{array}{l}\text { Control de la oxidación en } \\
\text { alimentos y conservador de } \\
\text { alimentos preparados. }\end{array}$ & $\begin{array}{l}\text { (Karadağ et al., 2019) (El-Desouky et al., } \\
\text { 2019) (Ali et al., 2019) (Sumintarti et al., } \\
\text { 2018). }\end{array}$ \\
\hline Ácido clorogénico. & $\begin{array}{l}\text { Antioxidante, nefroprotectora } \\
\mathrm{y} \text { anti infecciosa. }\end{array}$ & $\begin{array}{l}\text { Conservador de alimentos } \\
\text { preparados. }\end{array}$ & $\begin{array}{l}\text { (Ali et al., 2019) (de Oliveira et al., 2019) } \\
\text { (Gomes et al., 2020). }\end{array}$ \\
\hline Ácido $\rho$-cumárico. & Antibacteriana. & $\begin{array}{l}\text { Conservador de alimentos } \\
\text { preparados. }\end{array}$ & (Ali et al., 2019) (Gomes et al., 2020). \\
\hline$\alpha$-terpineno. & $\begin{array}{l}\text { Antioxidante y } \\
\text { antibacteriana. }\end{array}$ & $\begin{array}{l}\text { Control de la oxidación en } \\
\text { alimentos y conservador de } \\
\text { alimentos preparados. }\end{array}$ & $\begin{array}{l}\text { (Ali et al., 2019) (Kowalski et al., 2018) } \\
\text { (Ferreira et al., 2020). }\end{array}$ \\
\hline Geraniol. & Insecticida. & $\begin{array}{l}\text { Fumigante natural en el } \\
\text { control de plagas. }\end{array}$ & (Ali et al., 2019). \\
\hline Timol. & Insecticida. & $\begin{array}{l}\text { Fumigante natural en el } \\
\text { control de plagas. }\end{array}$ & $\begin{array}{l}\text { (Ali et al., 2019) (Kowalski et al., 2018) } \\
\text { (Ahsaei et al., 2019). }\end{array}$ \\
\hline Sabineno. & Antifúngica e insecticida. & $\begin{array}{l}\text { Fumigante natural en el } \\
\text { control de plagas. }\end{array}$ & (Ali et al., 2019) (Kowalski et al., 2018). \\
\hline$\alpha$-felandreno. & Antifúngica e insecticida. & $\begin{array}{l}\text { Fumigante natural en el } \\
\text { control de plagas. }\end{array}$ & $\begin{array}{l}\text { (Ali et al., 2019) (Kowalski et al., 2018) } \\
\text { (Ferreira et al., 2020) (Elbahnasawy et al., } \\
\text { 2019). }\end{array}$ \\
\hline Eugenol. & $\begin{array}{l}\text { Acaricida, antifúngica, } \\
\text { antiproliferativa, } \\
\text { antiinflamatoria y } \\
\text { antioxidante. }\end{array}$ & $\begin{array}{l}\text { Conservador de alimentos } \\
\text { preparados en el tratamiento y } \\
\text { control de la caspa. }\end{array}$ & (Ali et al., 2019) (de Oliveira et al., 2019). \\
\hline Carvacrol. & Insecticida. & $\begin{array}{l}\text { Fumigante natural en el } \\
\text { control de plagas. }\end{array}$ & $\begin{array}{l}\text { (Ali et al., 2019) (Kowalski et al., 2018) } \\
\text { (Ahsaei et al., 2019). }\end{array}$ \\
\hline$\delta$-3-careno. & Insecticida. & $\begin{array}{l}\text { Fumigante natural en el } \\
\text { control de plagas. }\end{array}$ & (Kowalski et al., 2018) (Ahsaei et al., 2019). \\
\hline
\end{tabular}


Tabla II. Características de las técnicas de extracción convencional y no convencional.

\begin{tabular}{|c|c|c|c|c|c|c|}
\hline & Técnica & Principio & Ventajas & Desventajas & Aplicaciones & Referencias \\
\hline \multirow{5}{*}{$\begin{array}{l}\text { C } \\
\text { o } \\
\text { n } \\
\text { v } \\
\text { e } \\
\text { n } \\
\text { c } \\
\text { i } \\
\text { o } \\
\text { n } \\
\text { a } \\
\text { l }\end{array}$} & $\begin{array}{l}\text { Prensado } \\
\text { mecánico. }\end{array}$ & $\begin{array}{l}\text { Destrucción } \\
\text { mecánica de } \\
\text { estructuras } \\
\text { vegetales que } \\
\text { libera componentes } \\
\text { de interés. } \\
\end{array}$ & $\begin{array}{l}\text { No requiere calor o } \\
\text { uso de disolventes. }\end{array}$ & $\begin{array}{l}\text { Técnica no selectiva } \\
\text { y uso de equipo que } \\
\text { aplique para altas } \\
\text { presiones. }\end{array}$ & $\begin{array}{l}\text { Extracción de } \\
\text { aceite esencial } \\
\text { y jugo. }\end{array}$ & $\begin{array}{l}\text { (Ventura et al., 2017) } \\
\text { (El-Hadary et al., } \\
\text { 2019) (Sik et al., 2020) } \\
\text { (Debebe et al., 2019) } \\
\text { (Zhang et al., 2018). }\end{array}$ \\
\hline & Maceración. & $\begin{array}{l}\text { Fenómeno de } \\
\text { transporte donde } \\
\text { los componentes de } \\
\text { la matriz de interés } \\
\text { son transferidos } \\
\text { al disolvente } \\
\text { utilizado. }\end{array}$ & $\begin{array}{l}\text { Uso de disolventes } \\
\text { de bajo costo, no } \\
\text { requiere equipo } \\
\text { especializado } \\
\text { ni tratamiento } \\
\text { previo de la } \\
\text { muestra y permite } \\
\text { la obtención de } \\
\text { componentes } \\
\text { termolábiles. }\end{array}$ & $\begin{array}{l}\text { Uso de disolventes } \\
\text { orgánicos tóxicos, } \\
\text { elevado tiempo de } \\
\text { extracción y no es } \\
\text { selectiva. }\end{array}$ & $\begin{array}{l}\text { Obtención } \\
\text { de extractos } \\
\text { de plantas } \\
\text { con actividad } \\
\text { biológica. }\end{array}$ & $\begin{array}{l}\text { (Pantoja et al., 2017) } \\
\text { (Putnik et al., 2017) } \\
\text { (Nieto et al., 2018) } \\
\text { (Zoral et al., 2018) } \\
\text { (Senanayake, 2018) } \\
\text { (Campo, Gélvez \& } \\
\text { Ayala, 2018) (Sik et al., } \\
\text { 2020) (Debebe } \text { et al., } \\
\text { 2019) (Ngo et al., 2017) } \\
\text { (Zhang et al., 2018) } \\
\text { (Moreira et al., 2019) } \\
\text { (Ali et al., 2019). }\end{array}$ \\
\hline & $\begin{array}{l}\text { Extracción } \\
\text { por reflujo. }\end{array}$ & $\begin{array}{l}\text { Migración de } \\
\text { componentes } \\
\text { de interés a } \\
\text { un disolvente } \\
\text { sometido a } \\
\text { calentamiento y a } \\
\text { volumen constante } \\
\text { mediante el reflujo. }\end{array}$ & $\begin{array}{l}\text { Uso de disolventes } \\
\text { de bajo costo y no } \\
\text { requiere equipo } \\
\text { especializado ni } \\
\text { tratamiento previo } \\
\text { de la muestra. }\end{array}$ & $\begin{array}{l}\text { Uso de elevadas } \\
\text { cantidades de } \\
\text { disolventes } \\
\text { orgánicos tóxicos, } \\
\text { elevado tiempo } \\
\text { de extracción, no } \\
\text { selectiva y daña } \\
\text { componentes } \\
\text { termolábiles. }\end{array}$ & $\begin{array}{l}\text { Obtención } \\
\text { de extractos } \\
\text { de plantas } \\
\text { con actividad } \\
\text { biológica. }\end{array}$ & \begin{tabular}{|l} 
(Zhang et al., 2018) \\
(Pantoja et al., 2017) \\
(Putnik et al., 2017) \\
(Nieto et al., 2018) \\
(Zoral et al., 2018) \\
(Senanayake, 2018) \\
(Campo et al., 2018) \\
(Ngo et al., 2017) (Sik et \\
al., 2020) (Debebe et al., \\
2019) (Ali et al., 2019).
\end{tabular} \\
\hline & Percolación. & $\begin{array}{l}\text { Se basa en el } \\
\text { principio de la } \\
\text { maceración, con } \\
\text { la diferencia de } \\
\text { mantener un } \\
\text { flujo continuo } \\
\text { de disolvente } \\
\text { nuevo, evitando su } \\
\text { saturación. }\end{array}$ & $\begin{array}{l}\text { Uso de disolventes } \\
\text { de bajo costo, no } \\
\text { requiere equipo } \\
\text { especializado o } \\
\text { tratamiento previo } \\
\text { de la muestra. }\end{array}$ & $\begin{array}{l}\text { Uso de elevadas } \\
\text { cantidades de } \\
\text { disolventes } \\
\text { orgánicos tóxicos, } \\
\text { elevado tiempo de } \\
\text { extracción y no es } \\
\text { selectiva. }\end{array}$ & $\begin{array}{l}\text { Obtención } \\
\text { de extractos } \\
\text { de plantas } \\
\text { con actividad } \\
\text { biológica. }\end{array}$ & $\begin{array}{l}\text { (Zhang et al., 2018) } \\
\text { (Pantoja } \text { et al., 2017) } \\
\text { (Putnik et al., 2017) } \\
\text { (Nieto et al., 2018) } \\
\text { (Zoral } \text { et al., 2018) } \\
\text { (Senanayake, 2018) } \\
\text { (Campo et al., 2018) } \\
\text { (Sik et al., 2020) } \\
\text { (Debebe } \text { et al., 2019). }\end{array}$ \\
\hline & Trituración. & $\begin{array}{l}\text { Destrucción } \\
\text { mecánica y } \\
\text { reducción del } \\
\text { tamaño del } \\
\text { material vegetal } \\
\text { con el fin de } \\
\text { obtener fluidos de } \\
\text { interés contenidos } \\
\text { en la muestra. }\end{array}$ & $\begin{array}{l}\text { No requiere el } \\
\text { uso de disolventes } \\
\text { o equipo } \\
\text { especializado ni } \\
\text { tratamiento previo } \\
\text { de la muestra. }\end{array}$ & $\begin{array}{l}\text { Baja eficiencia de } \\
\text { extracción, pobre } \\
\text { selectividad y } \\
\text { severas condiciones } \\
\text { de extracción. }\end{array}$ & $\begin{array}{l}\text { Extracción de } \\
\text { aceite esencial } \\
\text { y jugo. }\end{array}$ & $\begin{array}{l}\text { (Ventura et al., 2017) } \\
\text { (Zhang et al., 2018) } \\
\text { (Pantoja et al., 2017) } \\
\text { (Putnik et al., 2017) } \\
\text { (Nieto et al., 2018) } \\
\text { (Zoral et al., 2018) } \\
\text { (Senanayake, 2018) } \\
\text { (Campo et al., 2018) } \\
\text { (Ngo et al., 2017) (Sik et } \\
\text { al., 2020) (Debebe et al., } \\
\text { 2019). }\end{array}$ \\
\hline
\end{tabular}


Tabla II. Características de las técnicas de extracción convencional y no convencional (continuación).

\begin{tabular}{|c|c|c|c|c|c|c|}
\hline \multirow{4}{*}{$\begin{array}{l}\mathbf{N} \\
\mathbf{0} \\
\mathbf{c} \\
\mathbf{0} \\
\mathrm{n} \\
\mathrm{v} \\
\mathrm{e} \\
\mathrm{n} \\
\mathrm{c} \\
\mathbf{i} \\
\mathbf{0} \\
\mathrm{n} \\
\mathbf{a} \\
\mathbf{l}\end{array}$} & $\begin{array}{l}\text { Extracción } \\
\text { asistida } \\
\text { con fluidos } \\
\text { supercríticos. }\end{array}$ & $\begin{array}{l}\text { Uso de disolventes } \\
\text { a presiones y } \\
\text { temperaturas por } \\
\text { encima de su punto } \\
\text { crítico. }\end{array}$ & $\begin{array}{l}\text { Rápida, uso } \\
\text { reducido o nulo } \\
\text { de disolventes } \\
\text { orgánicos, } \\
\text { conserva } \\
\text { componentes } \\
\text { térmicamente } \\
\text { lábiles, selectiva, } \\
\text { recuperación y } \\
\text { recirculación del } \\
\text { disolvente. }\end{array}$ & $\begin{array}{l}\text { Elevados costos } \\
\text { de operación y } \\
\text { conocimientos } \\
\text { técnicos de las } \\
\text { propiedades de los } \\
\text { fluidos supercríticos. }\end{array}$ & $\begin{array}{l}\text { Obtención } \\
\text { de extractos } \\
\text { polares y no } \\
\text { polares de } \\
\text { las plantas } \\
\text { (pueden } \\
\text { ser sólidos } \\
\text { o líquidos) } \\
\text { así como } \\
\text { componentes } \\
\text { de interés } \\
\text { de flores } \\
\text { comestibles. }\end{array}$ & $\begin{array}{l}\text { (Quintana et al., 2019) } \\
\text { (Palacio, Arroyave, } \\
\text { Cardona, Hurtado } \\
\text { \& Martínez, 2018) } \\
\text { (Khaw et al., 2017) } \\
\text { (Senanayake, 2018) (Sik } \\
\text { et al., 2020) (Debebe } \\
\text { et al., 2019) (Putnik et } \\
\text { al., 2017) (Zhang et al., } \\
\text { 2018) (Pantoja et al., } \\
\text { 2017) (Zhang, Wen, } \\
\text { Zhang, Duan \& Ma, } \\
\text { 2020) (Zhao et al., 2019) } \\
\text { (Ali et al., 2019). }\end{array}$ \\
\hline & $\begin{array}{l}\text { Extracción } \\
\text { asistida por } \\
\text { microondas. }\end{array}$ & $\begin{array}{l}\text { Uso de radiaciones } \\
\text { electromagnéticas } \\
\text { a frecuencias } \\
\text { de } 300 \mathrm{MHz}- \\
300 \mathrm{GHz} \text {, con } \\
\text { longitudes de onda } \\
\text { de } 1 \mathrm{~cm} \text { a } 1 \mathrm{~m} . \\
\text { Aplicación de los } \\
\text { fenómenos de } \\
\text { rotación dipolar y } \\
\text { conducción iónica } \\
\text { para calentar la } \\
\text { muestra. }\end{array}$ & $\begin{array}{l}\text { Extracción rápida, } \\
\text { uso reducido de } \\
\text { disolvente, costos } \\
\text { adicionales bajos, } \\
\text { reducción de la } \\
\text { pérdida de los } \\
\text { componentes } \\
\text { extraídos. }\end{array}$ & $\begin{array}{l}\text { Uso de elevadas } \\
\text { temperaturas, } \\
\text { limitada a la cantidad } \\
\text { de la muestra, uso } \\
\text { de disolventes } \\
\text { con propiedades } \\
\text { dieléctricas y no es } \\
\text { selectiva. }\end{array}$ & $\begin{array}{l}\text { Extracción de } \\
\text { componentes } \\
\text { de interés de } \\
\text { las plantas } \\
\text { medicinales } \\
\text { y de flores } \\
\text { comestibles. }\end{array}$ & $\begin{array}{l}\text { (Altemimi et al., 2017) } \\
\text { (Quintana et al., 2019) } \\
\text { (Palacio et al., 2018) } \\
\text { (Flores 2017) (Sik et } \\
\text { al., 2020) (Debebe et } \\
\text { al., 2019) (Putnik et } \\
\text { al., 2017) (Jesus et al., } \\
\text { 2019) (Campo et al., } \\
\text { 2018) (Zhang, et al., } \\
\text { 2018) (Zhang, et al., } \\
\text { 2020) (Zhao, et al., } \\
\text { 2019) (Ali, et al., 2019). }\end{array}$ \\
\hline & $\begin{array}{l}\text { Extracción } \\
\text { asistida por } \\
\text { ultrasonido. }\end{array}$ & $\begin{array}{l}\text { Uso del fenómeno } \\
\text { de cavitación, } \\
\text { trabaja a } \\
\text { frecuencias } \\
\text { mayores a los } 20 \\
\text { KHz. }\end{array}$ & $\begin{array}{l}\text { Extracción rápida, } \\
\text { uso reducido } \\
\text { de disolvente, } \\
\text { costos adicionales } \\
\text { bajos y elevados } \\
\text { rendimientos de } \\
\text { extracción. }\end{array}$ & $\begin{array}{l}\text { Técnica no selectiva } \\
\text { y en tiempos muy } \\
\text { prolongados puede } \\
\text { dañar componentes } \\
\text { lábiles de interés. }\end{array}$ & $\begin{array}{l}\text { Procesamiento } \\
\text { de comida y } \\
\text { extracción de } \\
\text { componentes } \\
\text { bioactivos de } \\
\text { las plantas } \\
\text { y de flores } \\
\text { comestibles. }\end{array}$ & $\begin{array}{l}\text { (Altemimi et al., 2017) } \\
\text { (Quintana et al., 2019) } \\
\text { (Palacio et al., 2018) } \\
\text { (Flores 2017) (Sik et } \\
\text { al., 2020) (Debebe et } \\
\text { al., 2019) (Putnik et al., } \\
\text { 2017) (Campo et al., } \\
\text { 2018) (Zhang et al., } \\
\text { 2018) (Joshi \& Gogate, } \\
\text { 2020) (Zhang et al., } \\
\text { 2020) (Zhao et al., 2019) } \\
\text { (Ali et al., 2019). }\end{array}$ \\
\hline & $\begin{array}{l}\text { Extracción } \\
\text { asistida por } \\
\text { enzimas. }\end{array}$ & $\begin{array}{l}\text { Uso de enzimas } \\
\text { hidrolíticas de } \\
\text { la pared celular } \\
\text { que permite la } \\
\text { liberación de } \\
\text { componentes de } \\
\text { interés. }\end{array}$ & $\begin{array}{l}\text { Permite la } \\
\text { obtención de } \\
\text { componentes } \\
\text { termolábiles y } \\
\text { tiene elevados } \\
\text { porcentajes de } \\
\text { extracción. }\end{array}$ & $\begin{array}{l}\text { Requieren de } \\
\text { condiciones } \\
\text { específicas de } \\
\text { operación y la } \\
\text { aplicación de calor } \\
\text { puede inactivar las } \\
\text { enzimas. }\end{array}$ & $\begin{array}{l}\text { Obtención de } \\
\text { polisacáridos } \\
\text { de interés, } \\
\text { carotenoides y } \\
\text { licopeno de las } \\
\text { plantas. }\end{array}$ & $\begin{array}{l}\text { (Putnik et al., 2017) } \\
\text { (Sik et al., 2020) } \\
\text { (Zhang, et al., 2018) } \\
\text { (Ali, et al., 2019) } \\
\text { (Panda \& Manickam, } \\
\text { 2019). }\end{array}$ \\
\hline
\end{tabular}


Tabla II. Características de las técnicas de extracción convencional y no convencional (continuación).

\begin{tabular}{|c|c|c|c|c|c|c|}
\hline $\begin{array}{l}\mathbf{c} \\
\mathbf{o} \\
\mathbf{n} \\
\mathbf{v}\end{array}$ & $\begin{array}{l}\text { Extracción } \\
\text { asistida con } \\
\text { líquidos } \\
\text { iónicos. }\end{array}$ & $\begin{array}{l}\text { Uso de la } \\
\text { solvatación } \\
\text { de sales a } \\
\text { temperaturas } \\
\text { inferiores a los } \\
100^{\circ} \mathrm{C} \text {, lo } \\
\text { que facilita la } \\
\text { migración del } \\
\text { componente } \\
\text { de interés al } \\
\text { disolvente. }\end{array}$ & $\begin{array}{l}\text { Estabilidad } \\
\text { química, térmica } \\
\text { y electroquímica, } \\
\text { excelente } \\
\text { solvatación de } \\
\text { componentes } \\
\text { naturales y } \\
\text { sintéticos. }\end{array}$ & $\begin{array}{l}\text { Falta investigación } \\
\text { que demuestre que } \\
\text { son ambientalmente } \\
\text { inocuos. }\end{array}$ & $\begin{array}{l}\text { Extracción de } \\
\text { componentes } \\
\text { bioactivos } \\
\text { de biomasa, } \\
\text { así como la } \\
\text { extracción } \\
\text { de moléculas } \\
\text { de interés de } \\
\text { diferentes } \\
\text { plantas. }\end{array}$ & $\begin{array}{l}\text { (Putnik et al., 2017) } \\
\text { (Ventura } \text { et al., 2017) } \\
\text { (Debebe } \text { et al., 2019) } \\
\text { (de Faria et al., 2018) } \\
\text { (Strehmel, Strunk \& } \\
\text { Strehmel, 2017). }\end{array}$ \\
\hline $\begin{array}{l}\mathrm{e} \\
\mathrm{n} \\
\mathrm{c} \\
\mathrm{i} \\
\mathrm{o} \\
\mathrm{n} \\
\mathrm{a} \\
\mathrm{l}\end{array}$ & $\begin{array}{l}\text { Extracción } \\
\text { asistida por } \\
\text { campos } \\
\text { eléctricos } \\
\text { pulsantes. }\end{array}$ & $\begin{array}{l}\text { Hace uso de } \\
\text { campos eléctricos } \\
\text { pulsantes (con } \\
\text { voltajes de } 100 \\
\text { o } 200 \mathrm{~V} / \mathrm{cm} \\
\text { hasta } 80 \mathrm{kV} / \mathrm{cm} \text { ) } \\
\text { que destruyen } \\
\text { las membranas } \\
\text { estructurales } \\
\text { favoreciendo la } \\
\text { transferencia de } \\
\text { masa. }\end{array}$ & $\begin{array}{l}\text { No calienta el } \\
\text { material, no } \\
\text { requiere el uso } \\
\text { de químicos, } \\
\text { tiempos y uso de } \\
\text { energía reducido y } \\
\text { alta eficiencia de } \\
\text { extracción. }\end{array}$ & $\begin{array}{l}\text { La matriz debe tener } \\
\text { una resistividad } \\
\text { eléctrica elevada, si } \\
\text { los componentes que } \\
\text { se desean obtener } \\
\text { son enzimas, por } \\
\text { ejemplo, puede } \\
\text { inactivar algunas de } \\
\text { ellas. }\end{array}$ & $\begin{array}{l}\text { Extracción de } \\
\text { antioxidantes } \\
\text { de las plantas, } \\
\text { en procesos de } \\
\text { secado, corte, } \\
\text { congelado y } \\
\text { pasteurización } \\
\text { en frío de los } \\
\text { alimentos. }\end{array}$ & $\begin{array}{l}\text { (Putnik et al., 2017) } \\
\text { (Jesus et al., 2019) } \\
\text { (Debebe et al., 2019) } \\
\text { (Campo et al., 2018) } \\
\text { (Zhang et al., 2018) } \\
\text { (Zhang et al., 2020) } \\
\text { (Leonhardt et al., 2020) } \\
\text { (Zhao et al., 2019) (Ali } \\
\text { et al., 2019). }\end{array}$ \\
\hline
\end{tabular}

El aceite esencial de las plantas se obtiene por arrastre de vapor, incluida la hidrodestilación o procesos alternativos con el mismo principio (Borges et al., 2018). El aceite esencial del romero emite un intenso aroma a alcanfor, debido al elevado contenido de esta molécula, con un peso de $14.5 \%$ (Bilska et al., 2019). Una técnica convencional de obtención del aceite esencial del romero es utilizando sus hojas frescas con hidrodestilación tipo Clevenger (Pereira et. al., 2017), técnica, que se puede eficientar, asistida por microondas (Elyemni et al., 2019). Una variación de la anterior metodología, es usando hojas secas de romero molidas, y un proceso de extracción por hidrodifusión y gravedad con un equipo de microondas (Ferreira et al., 2020).

\section{Conclusiones}

El uso de las plantas, ha acompañado el desarrollo de la humanidad a través del tiempo, hasta convertirse no sólo en una fuente de alimento, sino también, en una forma de tratar enfermedades o malestares, gracias a sus propiedades medicinales.

De la gran diversidad de hierbas, que han sido usadas de forma tradicional en la mejora de la salud humana, destacan las pertenecientes a la familia Lamiaceae, que cuenta con una gran variedad de especies aromáticas, con potencial terapéutico. Diversas investigaciones actuales, han demostrado científicamente los efectos benéficos que estas producen, resaltando en ésta familia la especie Rosmarinus officinalis, planta que produce una amplia gama de metabolitos secundarios, responsables de diversas actividades biológicas que han permitido su aplicación en la medicina tradicional.

Aunque las metodologías convencionales, de extracción de metabolitos secundarios del romero, son ampliamente utilizadas hoy en día, el estudio en el laboratorio de las tecnologías emergentes no convencionales, ha logrado mejorar los porcentajes de extracción y la selectividad de los componentes aislados del romero, permitiendo el escalamiento industrial de algunas de ellas.

Hoy en día, las investigaciones centran su atención en la optimización de metodologías, que permitan la mejor extracción de los componentes bioactivos del romero, así como la identificación de sus metabolitos, para conocer el mecanismo de estas actividades y usarlo en nuevas y potenciales aplicaciones.

\section{Agradecimientos}

Se agradece a la Facultad de Ciencias Químicas, por las facilidades otorgadas para llevar a cabo el Posgrado en Ciencia y Tecnología Química como estudiante de tiempo parcial de Emmanuel Flores Villa, así como al apoyo proporcionado por el CONACYT a través del proyecto SEPCONACYT Ciencia Básica 2017-2018, CB2017-2018 A1S-44977. 


\section{REFERENCIAS}

Abbaszadeh, B., Layeghhaghighi, M., Azimi, R. \& Hadi, N. (2020). Improving water use efficiency through drought stress and using salicylic acid for proper production of Rosmarinus officinalis L. Industrial Crops \& Products, 144, 1-7. https://doi.org/10.1016/j.indcrop.2019.111893

Abo, M. M., Elsadek, M. F., Taha, A. E., Abd, M. M. \& ElSabrout, K. (2020). Effect of housing system and rosemary and cinnamon essential oils on layers performance, egg quality, haematological traits, blood chemistry, immunity, and antioxidant. Animal, 10, 1-16. DOI: 10.3390/ ani 10020245

Ahsaei, S. M., Rodríguez, S., Salgado, M., Cocero, M. J., Talebi-Jahromi, K. \& Amoabediny, G. (2019). Insecticidal activity of spray dried microencapsulated essential oils of Rosmarinus officinalis and Zataria multiflora against Tribolium confusum. Crops Protection, 128, 1-11. https:// doi.org/10.1016/j.cropro.2019.104996

Akhbari, M., Masoum, S., Aghababaei, F. \& Hamedi, S. (2018). Optimization of microwave assisted extraction of essential oils from Iranian Rosmarinus officinalis L. using RSM. Journal of Food Science and Technology, 55, 2197-2207. https://doi.org/10.1007/s13197-018-3137-7

Ali, A., Chua, B. L. \& Chow, Y. H. (2019). An insight into the extraction and fractionation technologies of the essential oils and bioactive compounds in Rosmarinus officinalis L.: Past, present and future. Trends in Analytical Chemistry, 188, 338-351. https://doi.org/10.1016/j.trac.2019.05.040

Altemimi, A., Lakhssassi, N., Baharlouei, A., Watson, D. G. \& Lightfoot, D. A. (2017). Phytochemicals: Extraction, Isolation, and Identification of Bioactive Compounds from Plant Extracts. Plants, 6, 1-23. DOI: 10.3390/ plants6040042

Amaral, G. P., Mizdal, C. R., Stefanello S. T., Mendez, A. S. L., Puntel, R. L., de Campos, M. M. A., Soares, F. A. A. \& Fachinetto, R. (2017). Antibacterial and antioxidant effects of Rosmarinus officinalis L. extract and its fractions. Journal of Traditional and Complementary Medicine, 9, 2, 1-10. https://doi.org/10.1016/j.jtcme.2017.10.006

Barbieri, J. B., Goltz, C., Cavalheiro, F. B., Toci, A. T., Igarashi-Mafra, L. \& Mafra, M. R. (2019). Deep eutectic solvents applied in the extraction and stabilization of rosemary (Rosmarinus officinalis L.) phenolic compounds. Industrial Crops \& Products, 144, 1-8. https://doi.org/10.1016/j.indcrop.2019.112049

Basheer, A. I. (2018). Effect of alcoholic extract of rosmarinus against some type of enterobacteriaceae. Tikrit Journal of Pure Science, 23, 18-21. DOI: http://dx.doi.org/10.25130/ tjps.23.2018.104

Beltrán, K. L., Déciga, M., Aguilar, H., González, M. E., Martínez, M. F., Ramírez, M. A., Rios, M. Y. \& López, F. J. (2017). Synergistic antinociceptive interaction of Syzygium aromaticum or Rosmarinus officinalis coadministered with ketorolac in rats. Biomedicine
\& Pharmacotherapy, 94, 858-864. http://dx.doi. org/10.1016/j.biopha.2017.07.166

Benelli, L. \& Oliveira, W. P. (2019). Fluidized bed coating of inert cores with a lipid-based system loaded with a polyphenol-rich Rosmarinus officinalis extract. Food and Bioproducts Processing, 114, 216-226. https://doi. org/10.1016/j.fbp.2019.01.004

Bilska, A., Kobus, J., Kmiecik, D., Danyluk, B., Kowalski, R., Szymanowska, D., Gramza, A. \& Szczepaniak, O. (2019). Cholinesterase inhibitory activity, antioxidative potential and microbial stability of innovative liver pâté fortified with rosemary extract (Rosmarinus officinalis). Electronic Journal of Biotechnology, 40, 22-29. https:// doi.org/10.1016/j.ejbt.2019.03.007

Borges, R. S., Sánchez, B. L., Matias, A. C., Keita, H. \& Tavares, J. C. (2018). Rosmarinus officinalis essential oil: A review of its phytochemistry, anti-inflammatory activity, and mechanisms of action involved. Journal of Ethnopharmacology, 229, 29-45. https://doi. org/10.1016/j.jep.2018.09.038

Bridi, H., de Loreto, S. A., Anders, M. \& Von Poser, G. L. (2020). Terpenes from Cantinoa (Lamiaceae) native to Rio Grande do Sul, South Brazil. Biochemical Systematics and Ecology, 89, 1-5. https://doi.org/10.1016/j. bse.2019.103992

Brown, N., John, J. A. \& Shahidi, F. (2019). Polyphenol composition and antioxidant potential of mint leaves. Food Production, Processing and Nutrition, 1, 1, 1-14. https://doi.org/10.1186/s43014-019-0001-8

Campo, Y., Gélvez, V. M. \& Ayala A. (2018). Ultrasonido en el procesamiento (homogenización, extracción y secado) de alimentos. Biotecnología en el Sector Agropecuario y Agroindustrial, 16, 102-113. DOI: http://dx.doi. org/10.18684/bsaa.v16n1.628

Debebe, W. D., Shimelis, A. E., Asfaw, D. H. \& Jong, B. E. (2019). Evaluation of Ultrasonic-Assisted Extraction of Moringa stenopetala Leaves on Bioactive Compounds and Antioxidant Effect. Food Technology and Biotechnology, 57, 1-24. DOI: 10.17113/ftb.57.01.19.5877

De Faria, E. L. P., Gomes, M. V., Cláudio, A. F. M., Freire, C. S. R., Silvestre, A. J. D. \& Freire, M. G. (2018). Extraction and recovery processes for cynaropicrin from Cynara cardunculus L. using aqueous solutions of surface-active ionic liquids. Biophysical Reviews, 10, 915-925. https:// doi.org/10.1007/s12551-017-0387-y

De Oliveira, J. R., Camargo, S. E.A. \& de Oliveira, L. D. (2019). Rosmarinus officinalis L. (rosemary) as therapeutic and prophylactic agent. Journal of Biomedical Science, 26, 1-22. https://doi.org/10.1186/s12929-019-0499-8

Ebrahimi, E., Haghjou, M., Nematollahi, A. \& Goudarzian, F. (2020). Effects of rosemary essential oil on growth performance and hematological parameters of young great sturgeon (Huso huso). Aquaculture, 521, 1-6. https://doi.org/10.1016/j.aquaculture.2019.734909 
Elbahnasawy, A. S., Valeeva, E. R., El-Sayed, E. M. \& Rakhimov, I. I. (2019). The impact of thyme and rosemary on prevention of osteoporosis in rats. Journal of Nutrition and Metabolism, 2019, 1-11. https://doi. org/10.1155/2019/1431384

El-Desouky, M. A., Mahmoud, M. H., Riad, B. Y. \& Taha, Y. M. (2019). Nephroprotective effect of green tea, rosmarinic acid and rosemary on N-diethylnitrosamine initiated and ferric nitrilotriacetate promoted acute renal toxicity in Wistar rats. Interdisciplinary Toxicology, 12, 98-110. DOI: 10.2478/intox-2019-0012

El-Hadary, A. E., Elsanhoty, R. M. \& Ramadan, M. F. (2019). In vivo protective effect of Rosmarinus officinalis oil against carbon tetrachloride $\left(\mathrm{CCl}_{4}\right)$-induced hepatotoxicity in rats. PharmaNutrition, 9, 1-7. https://doi.org/10.1016/j. phanu.2019.100151

Elyemni, M., Louaste, B., Nechad, I., Elkamli, T., Bouia, A., Taleb, M., Chaouch, M. \& Eloutassi, N. (2019). Extraction of essential oils of Rosmarinus officinalis L. by two different methods: Hydrodistillation and Microwave assisted hydrodistillation. The Scientific World Journal, 2019, 1-7. https://doi.org/10.1155/2019/3659432

Endo, E. H., Costa, G. M., Makimori, R. Y., Ueda-Nakamura, T., Nakamura, C. V. \& Dias, B. P. (2018). Anti-biofilm activity of Rosmarinus officinalis, Punica granatum and Tetradenia riparia against methicillin-resistant Staphylococcus aureus (MRSA) and synergic interaction with penicillin. Journal of Herbal Medicine, 14, 48-54. https://doi.org/10.1016/j.hermed.2018.07.001

Ferreira, D. F., Lucas, B. N., Voss, M., Santos, D., Mello, P. A., Wagner, R., Cravotto, G. \& Barin, J. S. (2020). Solventfree simultaneous extraction of volatile and non-volatile antioxidants from rosemary (Rosmarinus officinalis L.) by microwave hydrodiffusion and gravity. Industrial Crops \& Products, 145, 1-8. https://doi.org/10.1016/j. indcrop.2020.112094

Fidan, H., Stankov, S., Ivanova, T., Stoyanova, A., Damyanova, S. \& Ercisli, S. (2019). Characterization of aromatic compounds and antimicrobial properties of four spice essential oils from family Lamiaceae. Ukrainian Food Journal, 8, 227-238. DOI: 10.24263/2304-974X2019-8-2-3

Fikry, S., Khalil, N. \& Salama, O. (2019). Chemical profiling, biostatic and biocidal dynamics of Origanum vulgare L. essential oil. AMB Express, 9:41, 1-10. DOI: 10.1186/ s13568-019-0764-y

Flores, E. (2017). Extracción de antioxidants de las bayas del sauco(Sambucus nigra L. subs. peruviana) conultrasonido, microondas, enzimas y maceración para la obtención de zumos funcionales. Información Tecnológica, 28, 121132. DOI: $10.4067 / \mathrm{S} 0718-07642017000100012$

Fotso, T. G., Tofel, H. K., Abdou, J. P., Tchao, N., Zourmba, C. M., Adler, C. \& Nukenine, E. N. (2019). Control of Callosobruchus maculatus (Coleoptera: Chrysomelidae)
Using Fractionated Extracts from Cameroonian Hemizygia welwitschii (Lamiaceae) Leaf on Stored Vigna unguiculata (Fabales: Fabaceae). Journal of Insect Science, 22, 1-9. DOI: 10.1093/jisesa/iez029

García, P., Romero, M. J., Llanderal, A., Cermeño, P., Lao, M. T. \& Segura, M. L. (2019). Effects of Drought Stress on Biomass, Essential Oil Content, Nutritional Parameters, and Costs of Production in Six Lamiaceae Species. Water, 11, 1-12. DOI: 10.3390/w11030573

Gomes, P. G. C., Veloso, A. F., Maynard, I. F. N., Marques, M. N., de Souza R. L., Pereira, M. M., Soares, C. M. F. \& Lima, A. S. (2020). Integrative process to extract chlorophyll and purify rosmarinic acid from rosemary leaves (Rosmarinus officinalis). Journal of Chemical Technology and Biotechnology, 2020, 1-8. DOI: 10.1002/ jctb. 6343

Goudjil, M. B., Zighmi, S., Hamada, D., Mahcene, Z., Bencheikh, S. E. \& Lajel, S. (2020). Biological activities of essential oils extracted from Thymus capitatus (Lamiaceae). South African Journal of Botany, 128, 274282. DOI: 10.1016/j.sajb.2019.11.020

Gürbüz, P., Martínez, A., Pérez, C., Martínez, L., Göger, F. \& Ayran, Í. (2019). Potential anti-Alzheimer effects of selected Lamiaceae plants through polypharmacology on glycogen synthase kinase- $3 \beta, \beta$-secretase, and casein kinase $1 \delta$. Industrial Crops \& Products, 138, 1-6. https:// doi.org/10.1016/j.indcrop.2019.05.080

Hamidpour, R., Hamidpour, S. \& Elias G. (2017). Rosmarinus officinalis (Rosemary): A Novel Therapeutic Agent for Antioxidant, Antimicrobial, Anticancer, Antidiabetic, Antidepressant, Neuroprotective, Anti-Inflammatory and Anti-Obesity Treatment. Biomedical Journal of Scientific \& Technical Research, 1, 1-6. DOI: 10.26717/ BJSTR.2017.01.000371

Hosseinie, H., Bolourian, S., Yaghoubi, E. \& Ghanuni, E. (2018). Optimization of heat and ultrasound-assisted extraction of polyphenols from dried rosemary leaves using response surface methodology. Journal of Food Processing and Preservation, 42, 1-15. DOI: 10.1111/ jfpp. 13778.

Huang, M., Wang, H., Xu, X., Lu, X., Song, X. \& Zhou, G. (2020). Effects of nanoemulsion-based edible coatings with composite mixture of rosemary extract and $\varepsilon$-poly-Llysine on the shelf life of ready-to-eat carbonado chicken. Food Hydrocolloids, 102, 1-9. https://doi.org/10.1016/j. foodhyd.2019.105576

Jesus, M. S., Genisheva,Z., Romaní,A., Pereira, R. N., Teixeira, J. A. \& Domingues, L. (2019). Bioactive compounds recovery optimization from vine pruning residues using conventional heating and microwave-assisted extraction methods. Industrial Crops \& Products, 132, 99-110. https://doi.org/10.1016/j.indcrop.2019.01.070

Joshi, S. M. \& Gogate, P. R. (2020). Intensification of dilute acid hydrolysis of spent tea powder using ultrasound for 
enhanced production of reducing sugars. Ultrasonics - Sonochemistry, 61, 1-7. https://doi.org/10.1016/j. ultsonch.2019.104843

Karim, N., Khan, I., Abdelhalim, A., Abdel-Halim, H. \& Hanrahan, J. R. (2017). Molecular docking and antiamnesic effects of nepitrin isolated from Rosmarinus officinalis on scopolamine-induced memory impairment in mice. Biomedicine \& Pharmacotherapy, 96, 700-709. http://dx.doi.org/10.1016/j.biopha.2017.09.121

Khaw, K. Y., Parat, M. O., Shaw, P. N. \& Falconer, J. R. (2017). Solvent Supercritical Fluid Technologies to Extract Bioactive Compounds from Natural Sources: A Review. Molecules, 12, 1-22. DOI: 10.3390/molecules22071186

Karadă̆, A. E., Demirci, B., Çaşkurlu, A., Demirci, F., Okur, M. E., Orak, D., Sipahi, H. \& Başer, K. H. C. (2019). In vitro antibacterial, antioxidant, anti-inflammatory and analgesic evaluation of Rosmarinus officinalis L. flower extract fractions. South African Journal of Botany, 125, 214-220. https://doi.org/10.1016/j.sajb.2019.07.039

Karpiński, T. M. (2020). Essential Oils of Lamiaceae Family Plants as Antifungals. Biomolecules, 10, 1-35. DOI: 10.3390/biom10010103

Kowalski, R., Kowalska, G., Pankiewicz, U., Mazurek, A., Sujka, M., Włodatczyk-Stasiak, M. \& Kałwa, K. (2018). Effect of the method of rapeseed oil aromatisation with rosemary Rosmarinus officinalis L. on the content of volatile fraction. LWT-Food Science and Technology, 95, 40-46. https://doi.org/10.1016/j.lwt.2018.04.045

Lemjallad, L., Chabir, R., Kandri, Y., El Ghadraoui, L., Ouazzani, F. \& Errachidi, F. (2019). Improvement of Heliciculture by Three Medicinal Plants Belonging to the Lamiaceae Family. The Scientific World Journal, 2019, 1-7. https://doi.org/10.1155/2019/2630537

Leonhardt, L., Käferböck, A., Smetana, S., de Vos, R., Toepfl, S. \& Parniakov, O. (2020). Bio-refinery of Chlorella sorokiniana with pulsed electric field pretreatment. Bioresource Technology, 301, 1-8. https://doi. org/10.1016/j.biortech.2020.122743

Milevskaya, V. V., Prasad, S. \& Temerdashev, Z. A. (2019). Extraction and chromatographic determination of phenolic compounds from medicinal herbs in the Lamiaceae and Hypericaceae families: A review. Microchemical Journal, 145, 1036-1049. https://doi.org/10.1016/j. microc.2018.11.041

Moreira, S. A., Alexandre, E. M. C., Pintado, M. \& Saraiva J. A. (2019). Effect of emergent non-thermal extraction technologies on bioactive individual compounds profile from different plant materials. Food Research International, 115, 177-190. https://doi.org/10.1016/j. foodres.2018.08.046

Ngo, T. V., Scarlett, C. J., Bowyer, M. C., Ngo, P. D. \& Voung, Q. V. (2017). Impact of Different Extraction Solvents on Bioctive Compounds and Antioxidant Capacity from the Root of Salacia chinenesis L. Journal of Food Quality,
17, 1-8. https://doi.org/10.1155/2017/9305047

Nieto, G., Ros, G. \& Castillo, J. (2018). Antioxidant and Antimicrobial Properties of Rosemary (Rosmarinus officinalis, L.): A Review. Medicines, 5, 1-13. DOI: 10.3390/medicines5030098

Palacio, E., Arroyave, J. D., Cardona, M., Hurtado, J. H. \& Martínez, J. (2018). Extracción de glucosidos de Stevia Rebaudiana (Bertoni) a partir de tecnologías de extracción verdes. Revista de Investigación Agraria y Ambiental, 10, 43-56. DOI: https://doi.org/10.22490/21456453.2336

Panda, D. \& Manickam, S. (2019). Cavitation TechnologyThe Future of Greener Extraction Method: A Review on the Extraction of Natural Products and Process Intensification Mechanism and Perspectives. Applied Sciences, 9, 1-26. DOI: 10.3390/app9040766

Pantoja, A. L., Hurtado, A. M. \& Martínez, H. A. (2017). Caracterización de aceite de semillas de maracuyá (Passiflora edulis Sims.) procedentes de residuos agroindustriales obtenido con $\mathrm{CO}_{2}$ supercrítico. Agroindustria y Ciencia de los Alimentos, 66, 178-185. http://dx.doi.org/10.15446/acag.v66n2.57786

Pereira, P. S., Maia, A. J., Tintino, S. R., Oliveira-Tintino, C. D. de M., Raulino, I. S. de S., Vega, M. C., Rolón, M., Coronel, C., Barros, L. M., Duarte, A. E., Menezes, I. R. A., Coutinho, H. D. M. \& da Silva, T. G. (2017). Trypanocide, antileishmania and cytotoxic activities of the essential oil from Rosmarinus officinalis, L. in vitro. Industrial Crops \& Products, 109, 724-729. http://dx.doi. org/10.1016/j.indcrop.2017.09.030

Putnik, P., Bursać, D., Režek, A., Barba, F. J., Cravotto, G., Binello, A., Lorenzo, J. M. \& Shpigelman, A. (2017). Innovative "Green" and Novel Strategies for the Extraction of Bioactive Added Value Compunds from Citrus Wastes-A Review. Molecules, 22, 1-24. DOI: 10.3390/molecules22050680

Quintana, S. E., Villanueva, D., Reglero, G., García, M. R. \& Fornari, T. (2019). Supercritical antisolvent particle precipitation and fractionation of rosemary (Rosmarinus officinalis L.) extracts. Journal of $\mathrm{CO}_{2}$ Utilization, 34, 479-489. https://doi.org/10.1016/j.jcou.2019.07.032

Risaliti, L., Kehagia, A., Daoultzi, E., Lazari, D., Bergonzi, M. C., Vergkizi-Nikolakaki, S., Hadjipavlou-Litina, D. \& Bilia, A. R. (2019). Liposomes loaded with Salvia triloba and Rosmarinus officinalis essential oils: In vitro assessment of antioxidant, antiinflamatory and antibacterial activities. Journal of Drug Delivery Science and Technology, 51, 493-498. https://doi.org/10.1016/j. jddst.2019.03.034

Romero, C., Ramasamy, K., Meng, S., Abdul, A. B. \& Agatonovic-Kustrin, S. (2019). HPTLC based approach for bioassay-guided evaluation of antidiabetic and neuroprotective effects of eight essential oils of the Lamiaceae family plants. Journal of Pharmaceutical and Biomedical Analysis, 178, 1-7. https://doi.org/10.1016/j. 
jpba.2019.112909

Ruzzene, S., Sete, R. M., Botelho, E. L. \& Alberton, O. (2019). Meta-analysis of Lamiaceae and Euphorbiaceae medicinal plants inoculated with Arbuscular mycorrhizal fungi. Australian Journal of Crops Science, 13, 588-598. doi: 10.21475/ajcs.19.13.04.p1615

Said, A. M., Waheed, R. M. \& Khalifa, O. A. (2019). Protective Role of Rosemary Ethanolic Extract on Thioacetamide Induced Hepatic Encephalopathy: Biochemical and Molecular Studies. Australian Journal of Basic and Applied Sciences, 13, 1-6. DOI: 10.22587/ ajbas.2019.13.4.1

Sadeh, D., Nitzan, N., Chaimovitsh, D., Shachter, A., Ghanim, M. \& Dudai, N. (2019). Interactive effects of genotype, seasonality and extraction method on chemical compositions and yield of essential oil from rosemary (Rosmarinus officinalis L.). Industrial Crops \& Products, 138, 1-7. https://doi.org/10.1016/j.indcrop.2019.05.068

Selmi, S., Rtibi, K., Grami, D., Sebai, H. \& Marzouki, L. (2017). Rosemary (Rosmarinus officinalis) essential oil components exhibit anti-hyperglycemic, antihyperlipidemic and antioxidant effects in experimental diabetes. Pathophysiology, 24, 297-303. http://dx.doi. org/10.1016/j.pathophys.2017.08.002

Senanayake, N. (2018). Rosemary extract as a natural source of bioactive compounds. Journal of Food Bioactives, 2, 51-57. DOI: $10.31665 / J F B .2018 .2140$

Shanaida, M. \& Golembiovska, O. (2018). Identification and component analysis of triterpenoids in Monarda fistulosa L. and Ocimum americanum L. (Lamiaceae) aerial parts. Pharmaceutical Science, 13, 26-31. DOI: 10.15587/25194852.2018.135767

Sik, B., Hanczné, E. L., Kapcsándi, V. \& Ajtony, Z. (2020). Conventional and nonconventional extraction techniques for optimal extraction processes of rosmarinic acid from six Lamiaceae plants as determined by HPLCDAD measurement. Journal of Pharmaceutical and Biomedical Analysis, 184, 1-8. https://doi.org/10.1016/j. jpba.2020.113173

Sik, B., Kapcsándi, V., Székelyhidi, R., Hanczné, E. L. \& Ajtony, Z. (2019). Recent Advances in the Analysis of Rosmarinic Acid From Herbs in the Lamiaceae Family. Natural Product Communications, 2019, 1-10. DOI: 10.1177/1934578X19864216

Strehmel, N., Strunk, D. \& Strehmel, V. (2017). Extraction of natural products from bark of Betula pendula using ionic liquids. Metabolomics, 13:135, 1-8. DOI: 10.1007/ s11306-017-1271-2

Sullbarán, A., Matiz, G. E. \& Baena, Y. (2018). Acetilación del almidón de millo (Pennisetum glaucum) y evaluación de su aplicación como posible excipiente. Revista Colombiana de Ciencias Químicas y Farmacia, 47, 255276. http://dx.doi.org/10.15446/rcciquifa.v47n2.73969
Sumintarti, Fatimasari, N., Hajrah-Yusuf, A. \& Ruslin, M. (2018). Effects of rosemary (Rosmarinus officinalis L.) leaf extract on angular cheilitis induced by Staphylococcus aureus and Candida albicans in male wistar rats. International Journal of Applied Pharmaceutics, 10, 178181. DOI: http://dx.doi.org/10.22159/ijap.2018.v10s1.39

Tančinová, D., Medo, J., Mašková, Z., Foltinová, D. \& Árvay, J. (2019). Effect of essential oils of Lamiaceae plants on the Penicillium commune. Journal of Microbiology, Biotechnology and Food Sciences, 8, 1111-1117. DOI: 10.15414/jmbfs.2019.8.4.1111-1117

Trupti, P. \& Gadekar, S. S. (2018). To study the antidandruff activity of rosemary oil, basil oil, coleus oil over selenium sulfide. Journal of Pharmaceutical and BioSciences, $\mathbf{6}$, 36-39. DOI: $10.31555 / \mathrm{jpbs} / 2018 / 6 / 2 / 36-39$

Ventura, S. P. M., Silva, F. A., Quental, M. V., Mondal, D., Freire, M. G. \& Coutinho, J. A. P. (2017). Ionic-LiquidMediated Extraction and Separation Processes for Bioactive Compounds: Past, Present and Future Trends. ACS Publications, 117, 6984-7052. DOI: 10.1021/acs. chemrev.6b00550

Zhang, J., Chen, T., Li, K., Xu, H., Liang, R., Wang, W., Li, H., Shao, A. \& Yang, B. (2019). Screening active ingredients of rosemary based on spectrum-effect relationships between UPLC fingerprint and vasorelaxant actitivity using three chemometrics. Journal of Chromatography B, 1134-1135, 1-8. https://doi.org/10.1016/j.jchromb.2019.121854

Zhang, J., Wen, C., Zhang, H., Duan, Y. \& Ma, H. (2020). Recent advances in the extraction of bioactive compounds with subcritical water: A review. Trends in Food Science \& Technology, 95, 183-195. https://doi.org/10.1016/j. tifs.2019.11.018

Zhang, Q. W., Lin, L. G. \& Ye, W. C. (2018). Techniques for extraction and isolation of natural products: a comprehensive review. Chinese Medicine, 13(20), 1-26. https://doi.org/10.1186/s13020-018-0177-x

Zhao, L., Fan, H., Zhang, M., Chitrakar, B., Bhandari, B. \& Wang, B. (2019). Edible flowers: Review of flower processing and extraction of bioactive compounds by novel technologies. Food Research International, 126, 1-14. https://doi.org/10.1016/j.foodres.2019.108660

Zinicovscaia, I., Gundorina, S., Vergel, K., Grozdov, D., Ciocarlan, A., Aricu, A., Dragalin, I. \& Ciocarlan, N. (2020). Elemental analysis of Lamiaceae medicinal and aromatic plants growing in the Republic of Maldova using neutron activation analysis. Phytochemistry Letters, 35, 119-127. https://doi.org/10.1016/j.phytol.2019.10.009

Zoral, M.A., Ishikawa, Y., Ohshima, T., Futami, K., Endo, M., Maita, M. \& Katagiri, T. (2018). Toxicological effects and pharmacokinetics of rosemary (Rosmarinus officinalis) extract in common carp (Cyprinus carpio). Aquaculture, 495, 955-960. https://doi.org/10.1016/j. aquaculture.2018.06.048 